\title{
QUELQUES FACTEURS DE VARIATION DU TEMPS DE COAGULATION DES LAITS INDIVIDUELS DE VACHES
}

\author{
P. AURIOI, \\ Avec la collaboration technique de M. Dupont et Marie-Thérèse Le Borgne \\ Station de Recherches sur l'Elevage, \\ Centre national de Recherches zootechniques, Jouy-en-Josas.
}

\begin{abstract}
SOMMAIRE
A partir du contrôle hebdomadaire des laits produits au cours de 107 lactations (63 lactations de vaches normandes et 44 de vaches françaises frisonnes pie-noire (FFPN)), nous avons étudié l'influence de différents facteurs sur le temps de coagulation du lait sous l'action de la présure.

Les principaux résultats obtenus sont les suivants :

- Les génisses présentent des temps de coagulation légèrement inférieurs à ceux des vaches en deuxième et troisième lactation.

- Le stade de lactation agit nettement sur le temps de coagulation qui, après une phase d'augmentation s'étalant sur les 200 premiers jours de lactation, passe par un maximum, peu prononcé, pour décroître ensuite légèrement pendant les derniers ıoo jours de lactation. Au total, le temps de coagulation varie en moyenne de 75 à II 5 p. Ioo par rapport à sa valeur moyenne au cours de l'ensemble de la lactation.

- L'évolution du temps de coagulation avec le stade de lactation semble être liée en grande partie à l'évolution du rapport calcium / azote dans le lait.

- La race, et encore plus l'individu, constituent d'importantes sources de variation : le temps de coagulation moyen au cours de la lactation est de $16,6 \pm 0,5 \mathrm{mn}$ pour la race normande contre $20,0 \pm 0,6 \mathrm{mn}$ pour la race FFPN. Dans le même troupeau, les coefficients de variation des temps de coagulation moyens sont très élevés : 24,8 p. I00 pour les vaches normandes et I 8,7 p. 100 pour les vaches FFPN.

—L'analyse des résultats obtenus sur 8 paires de jumelles univitellines et 8 paires de jumelles bivitellines montre clairement que le caractère " vitesse de coagulation du lait " est en partie sous la dépendance de facteurs génétiques. Étant donné la grande variabilité de ce caractère, il doit être possible, le cas échéant, de l'améliorer par sélection.
\end{abstract}

Le phénomène de la coagulation du lait sous l'action de la présure a fait l'objet de nombreuses études, mais presque toutes ont été envisagées sous l'angle chimique ou physicochimique. Notre intention est, ici, de rechercher si l'aptitude du lait à la coagulation peut être, au même titre que divers autres caractères, améliorée par sélection.

Ce problème revêt une certaine importance, car un comportement normal visà-vis de la présure constitue une qualité essentielle pour tous les laits destinés à la production de fromage. 
Or, depuis les travaux de Koestier (I925), McDowal, L, Dol,by et McDowel, (I937), Peitola (I949), Mocouot, Alais et Chevalier (I954), nous savons que les laits individuels peuvent présenter de grandes variations dans leur aptitude à la coagulation par la présure. D'après Mocouot, Alais et ChevaliER (I954), la fréquence des laits individuels anormaux quant à ce caractère serait de I à 2 p. Ioo dans la race pierouge de 1'Est et de ro à II p. Ioo dans la race Salers. Ces chiffres s'entendent après élimination des cas de mammites. En Finlande, c'est 46 p. Ioo des laits individuels qui sont considérés comme anormalement "lents " par PELTOLA (I957) ; il est vrai que cet auteur a englobé dans son étude des laits de fin de lactation, qui sont "lents », dans $25 \mathrm{p}$. Ioo des cas lorsque la production laitière par traite est comprise entre 2 et $3 \mathrm{~kg}$, dans $70 \mathrm{p}$. Ioo des cas quand elle est inférieure à I $\mathrm{kg}$.

KOESTLER (I925), en Suisse, avait détecté I3 p. IOO de laits "lents".

Avant d'examiner les possibilités d'une sélection portant sur ce caractère, nous analyserons les différentes causes de variation, d'origine génétique ou non.

\section{MÉTHODES ET MATÉRIEL}

\section{Mesure DU TEMPS DE COAgulation}

Nous avons utilisé l'épreuve de coagulation du lait par la présure inspirée de DAvis et MC CLEMONT (1939) ; nous pouvons la résumer brièvement ainsi : à Io $\mathrm{ml}$ de lait de chaque échantillon, on ajoute I ml d'une solution de présure commerciale au. I/10 000 (additionnée de 0,04 p. 100 de pourpre de bromocrésol), le taux de dilution étant choisi de manière à obtenir une durée de coagulation, ou temps de coagulation, de I 5 à $\mathrm{I} 8 \mathrm{mn}$ à $35^{\circ} \mathrm{C}$ pour un lait de mélange moyen.

Le temps de coagulation ( $\left.t_{c}\right)$ est représenté par l'intervalle qui sépare l'instant de l'emprésurage de l'instant où les premiers flocons apparaissent sur la paroi du tube. Nous l'avons exprimé en minutes et $\mathrm{I} / \mathrm{IO}^{\mathrm{e}}$ de minute.

Les échantillons ont été examinés dans les quelques heures suivant la traite : ce dernier point est très important car on sait que, sur des échantillons de lait conservés à la température ambiante, une modification même légère du $\mathrm{pH}$ change considérablement la durée de coagulation du lait. D'autre part, la conservation des échantillons en glacière pendant I 2 à I 5 heures, aux environs de $+3^{\circ} \mathrm{C}$, augmente le temps de coagulation (Ling, i937, Mocouot, Alats et Chevaliter, 1954, Peltola et VoGT, 1959).

Le lait des vaches contrôlées a subi un examen bactériologique détaillé destiné à détecter les mammites. Les rares cas de laits mammiteux ont été éliminés de notre étude.

Nous avons testé la précision de la méthode utilisée au cours d'essais portant sur I 80 répétitions, effectuées par le même opérateur, à 45-75 minutes d'intervalle. La corrélation obtenue entre deux lectures successives est presque parfaite $(r=+0,987)$. Il n'y a donc pas de différence significative entre deux mesures consécutives prises sur le même lait, même à 75 minutes d'intervaile.

\section{MATÉRIEL ANIMAL ET ÉCHANTILLONS DE LAITS}

Cette étude a porté sur toutes les vaches du troupeau du C. N. R. Z. qui sont entrées en lactation entre novembre $195^{6}$ et mars $195^{8}$. Nous n'avons prélevé d'échantillons que sur les laits de la traite du matin, pour pouvoir effectuer les mesures rapidement après la récolte du lait. Les vaches ont été contrôlées une fois par semaine au cours de leur lactation.

Nos résultats concernent Io7 lactations (44 lactations de vaches françaises frisonnes pie-noire (FFPN) et $6_{3}$ lactations de vaches normandes $(\mathrm{N})$.

Afin de préciser certains points particuliers, nous avons également utilisé 750 lactations de vaches pie-rouge de l'Est, contrôlées, une fois par mois seulement, dans près de 200 élevages du Jura (AURIOL, I954, AURIOL et MocQuot, 1957). Pour ces lactations, nous avons disposé, non seulement des $t_{c}$ déterminés à l'étable sur les laits frais $\left({ }^{*}\right)$, mais aussi des résultats des dosages mensuels de calcium (JENNESs, I953) et d'azote (Kjeldahl) effectués sur les mêmes laits par la sta. tion de Recherches laitières de Poligny ${ }^{* *}$;

(*) Par les contrôleurs du Syndicat de contrôle laitier du Jura.

$\left.{ }^{* *}\right)$ Ces dosages ont été effectués par MM. E. BLANC-PATin et R. Jeunet, Station de Recherches laitières de Poligny. 
Nous avons calculé pour chaque lactation le temps de coagulation moyen, moyenne arithmétique des $\mathrm{t}_{\boldsymbol{c}}$ hebdomadaires.

\section{PRINCIPAUX RÉSULTATS}

Infuence du numéro de lactation et de la saison de vêlage.

Ces deux facteurs n'ont pu faire l'objet que d'une étude préliminaire, étant donné le petit nombre de lactations dont nous disposions. Les quelques résultats qui figurent ci-dessous montrent toutefois que les génisses ont tendance à produire des laits coagulant un peu plus rapidement que ceux des vaches en deuxième ou troisième lactation :

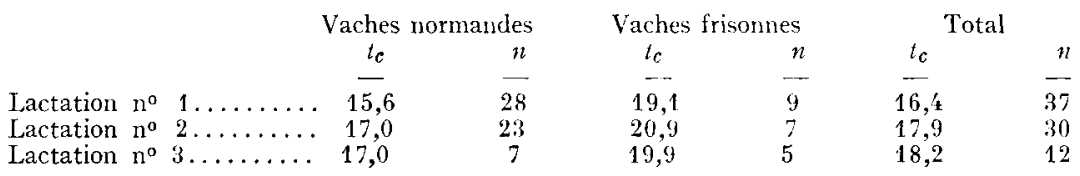

En ce qui concerne l'influence de la saison de vêlage, nos données sont encore plus fragmentaires. Nous avons noté cependant une tendance à l'augmentation du $t_{c} \mathrm{chez}$ les vaches ayant vêlé en été et une tendance à la diminution pour les vêlages d'hiver.

\section{Influence du stade de lactation.}

D'après McDowal, Dor,by et McDowel, (I937), le $t_{c}$ irait en augmentant progressivement au fur et à mesure qu'on s'éloigne du vêlage, cette augmentation étant d'autant plus rapide qu'on se trouve plus près de la fin de lactation. Les auteurs précédents signalent également que l'allure de la courbe d'évolution du $t_{c}$ avec le stade de lactation dépend en partie de la race : la pente de cette courbe est de plus en plus grande quand on passe de la Jersiaise à la Frisonne et surtout à la race Ayrshire.

Par contre, Peitora (I949), Mocquot, Alais et CheVAlier (I954) observent que les $t_{c}$ sont relativement constants, sauf en début et fin de lactation où ils prennent des valeurs plus faibles.

Nos données viennent corroborer en partie ces dernières observations : le $t_{c}$ augmente assez rapidement en début de lactation, moins vite par la suite, mais cette augmentation se poursuit jusqu'aux environs du septième mois de lactation.

Contrairement aux observations de McDowal, Dol,By et McDowel, (I937), qui relèvent un accroissement plus prononcé en fin de lactation, nous constatons plutôt une tendance à la diminution du $t_{c}$, durant le dernier tiers de la lactation (cf. fig.I).

En général, les laits récoltés pendant le dernier tiers de la lactation coagulent donc plus difficilement que ceux recueillis pendant les deux premiers tiers de la lactation.

Le profil de la courbe du $t_{c}$ varie beaucoup d'un individu à l'autre et les conclusions précédentes ne sont valables que pour une moyenne d'individus. De plus, dans les premiers jours de lactation et pendant la période de tarissement, les laits coagulent souvent de façon très irrégulière d'un contrôle au suivant et nous avons préféré ne pas en tenir compte.

Les vaches normandes et françaises frisonnes pie-noire se comportent dans 1'ensemble d'une façon analogue. Notons toutefois que le $t_{c}$ semble augmenter légèrement 


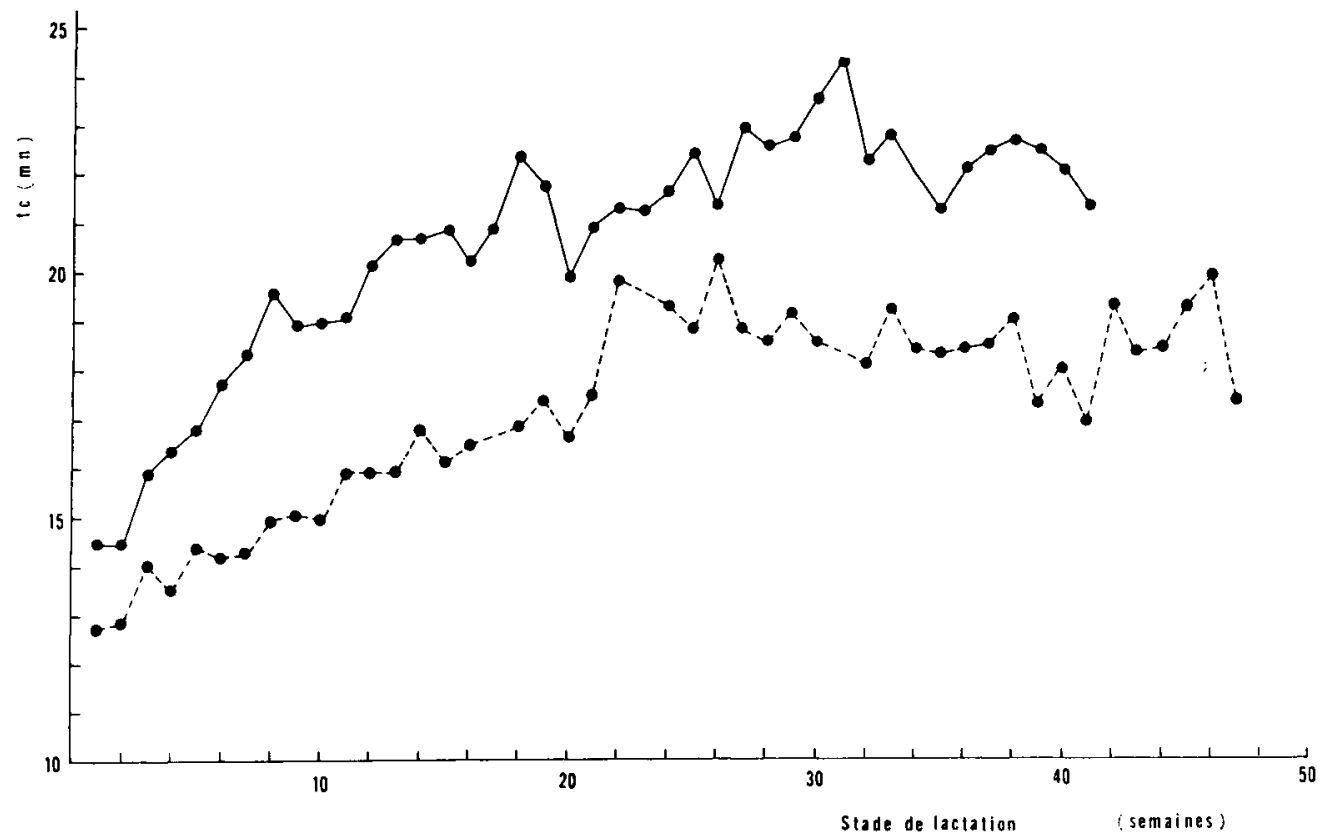

FIG. I - Évolution dn temps de coagulation avec le stade de lactation.

Vaches normandes.

Vaches françaises frisonnes pie-noire.

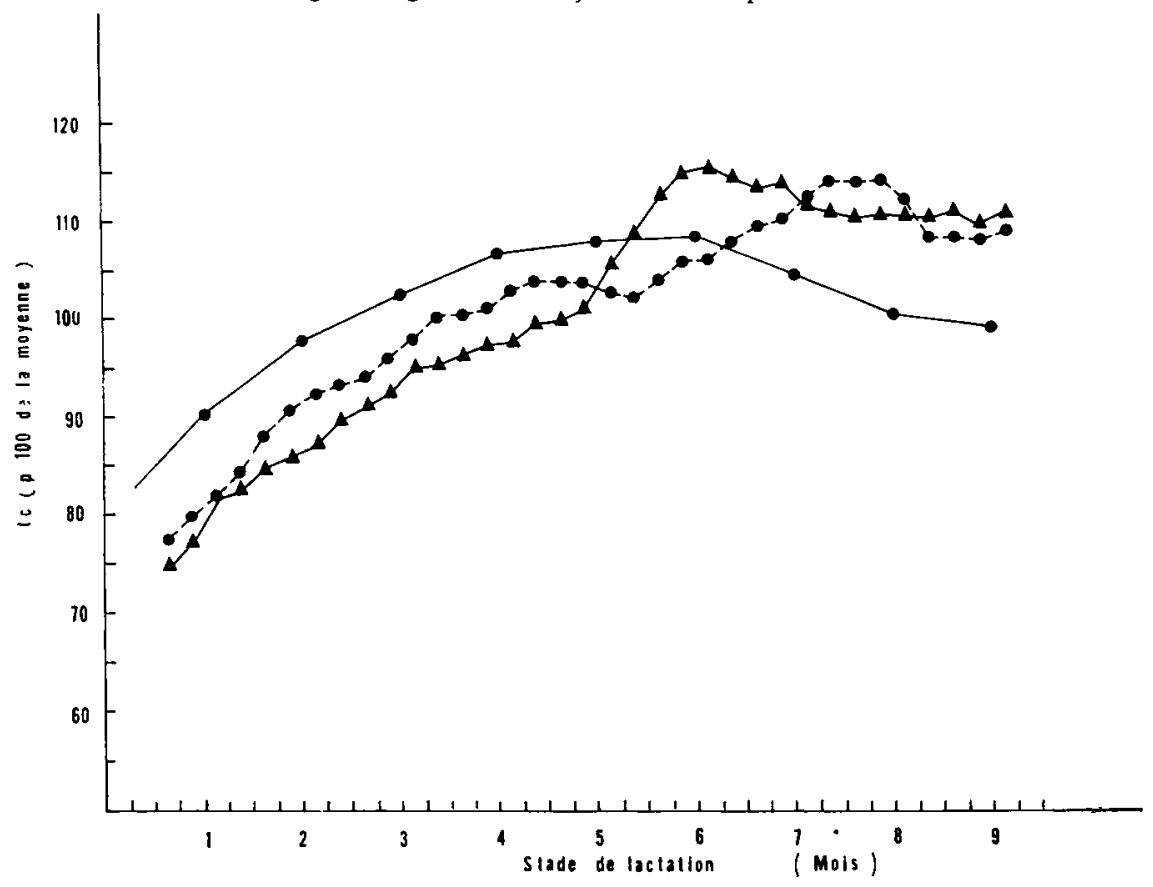

FIG. 2 - Evolution du temps de coagulation avec le stade de lactation.

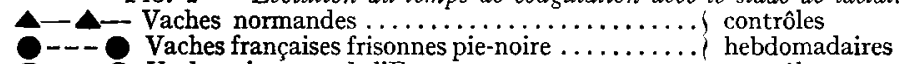

Vaches pie-rouge de l'Est . . . ....................... contrôles mensuels

Les $t_{c}$ ont exprimés en $p$. Ioo du $t_{c}$ moyen observé pour chacune des races. Dans le cas des vaches normandes et frisonnes, les courbes ont été ajustées par la méthode des moyennes mobiles. 
plus vite en début de lactation - et pendant un peu plus longtemps - chez la FFPN. Ce décalage n'est pas dû̀ à une différence dans l'intervalle moyen vêlagefécondation, puisque ce dernier est de $67 \mathrm{j}$ pour les FFPN et de $72 \mathrm{j}$ pour les normandes. De nouvelles données seront nécessaires pour éclaircir ce point.

L'étude comparative de l'évolution du $t_{c}$, au cours de la lactation, pour les différentes races, est plus facile à faire sur la figure 2 , où les $t_{c}$ sont exprimés en $p$. Ioo de la valeur moyenne observée sur l'ensemble de la lactation. Sur ce graphique figure également la courbe correspondant aux $75^{\circ}$ lactations de vaches pie-rouge de 1'Est, contrôlées une fois par mois seulement dans le Jura.

La courbe correspondant à la race pie-rouge de l'Est est du même type que celles obtenties pour les Frisonnes et les normandes du Centre National de Recherches zootechniques. Notons cependant que le $t_{c}$ semble moins varier au cours de la lactation chez les vaches pie-rouge. De plus, il diminuerait plus rapidement pendant le dernier tiers de la lactation.

Quels sont les facteurs qui commandent cette évolution au cours de la lactation?

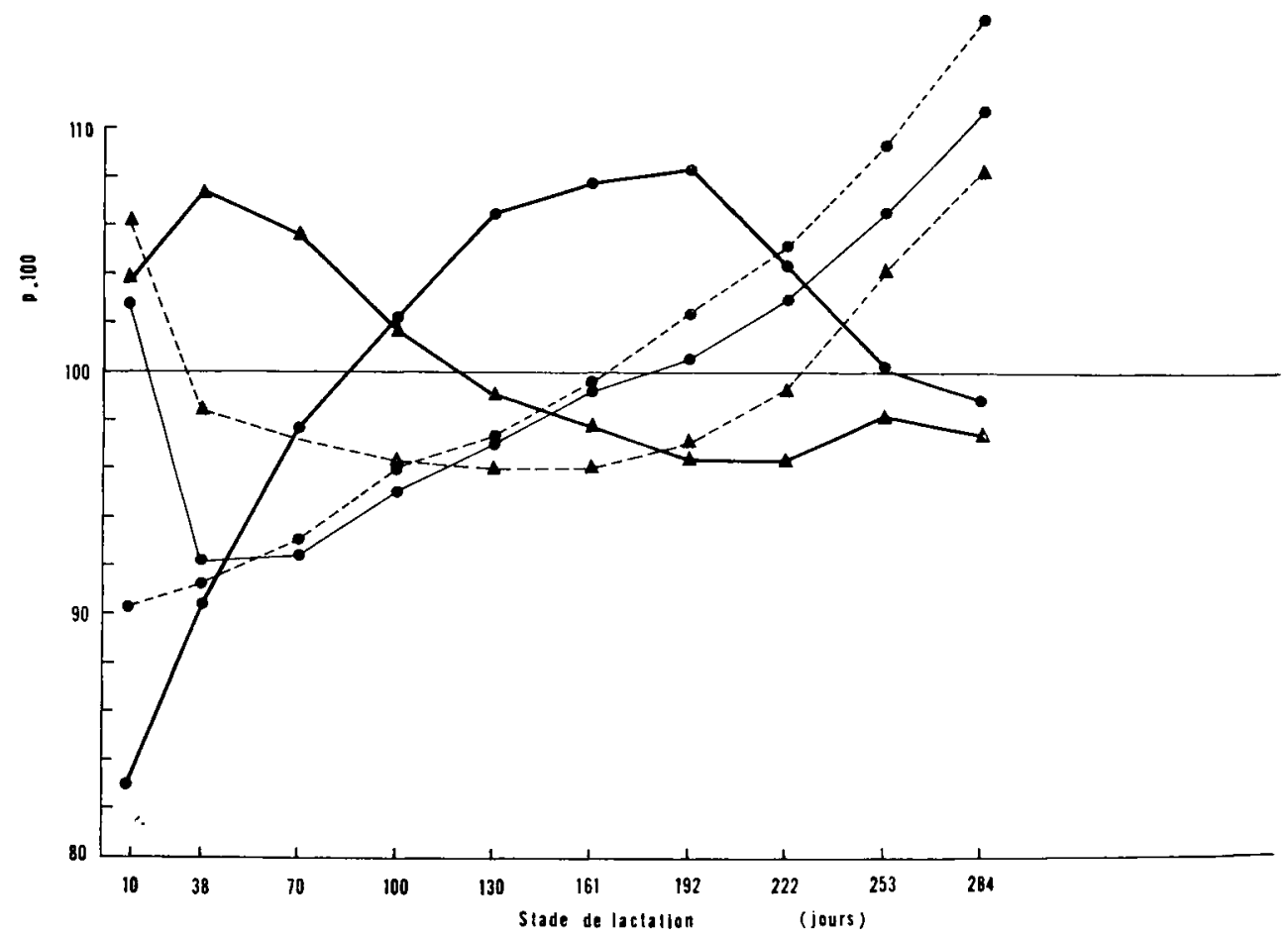

FIG. 3 - Relation, au cours de la lactation, entre temps de coagulation el quelques consituants du lait (vaches pie-rouge de l'Est).

Les valeurs prises au cours de la lactation par les différents constituants du lait ont été exprimées en p. Ioo de la valeur moyenne observée pour chacun d'eux sur l'ensemble de la lactration.

Étant donné le rôle joué par le calcium dans le phéncmène de coagulation du lait par la présure, il est logique de rapprocher les valeurs observées pour le $t_{c}$ de celles obtenues simultanément pour le rapport $\mathrm{Ca} / \mathrm{N}$ : d'une façon générale, on sait que plus 
ce rapport est élevé, plus le lait coagule rapidement. Pour les vaches suivies au Centre National de Recherches zootechniques, nous ne disposons pas des valeurs du rapport $\mathrm{Ca} / \mathrm{N}$. Aussi est-ce à partir des données du Jura (AURIor, I957) que nous avons abordé ce problème. La figure 3 montre clairement que les laits les plus "lents » correspondent aux valeurs les plus faibles du rapport $\mathrm{Ca} / \mathrm{N}$. Fin début et fin de lactation, la relation est moins nette, ce qui peut s'expliquer en partie par les réactions irrégulières du lait à ces stades.

De plus, nous constatons que ce sont bien les variations du rapport $\mathrm{Ca} / \mathrm{N}$ qui présentent la meilleure relation avec celles du $t_{c}$, comme l'ont du reste déjà signalé Mocquot, Alais et Chevalier (r954).

\section{Influence de la race:}

La figure I fait apparaître nettement des différences entre normandes (N) et frisonnes (FFPN) lorsque les $t_{c}$ sont considérés au même stade de la lactation. Ces différences se traduisent, au niveau des $t_{c}$ moyens sur l'ensemble d'une lactation, par un écart de $3,4 \mathrm{mn}$, hautement significatif $(\mathrm{P}<0, \mathrm{or})$. Le tableau I montre que le lait produit par les vaches normandes coagule plus rapidement que celui des vaches Frisonnes ( 16,6 contre $20,0 \mathrm{mn}$ ).

Nous n'avons pu faire la comparaison avec la race pie-rouge de 1'Eist, car, pour des raisons de facilité d'exécution, la concentration de la solution de présure utilisée pout l'épreuve de coagulation était beaucoup plus grande que celle employée au Centre National de Recherches zootechniques.

\section{Inffuence de l'individu - facteurs génétiques.}

A notre connaissance, le seul résultat publié concernant le déterminisme héréditaire du $t_{c}$ est celui de Per,Tol A (I957) ; cet auteur, après examen de 20 couples mère-fille provenant, il est vrai, de taureaux différents, conclut en faveur de l'action de facteurs génétiques.

Les différences, très nettes, que nous avons nous-mêmes observées entre deux races exploitées dans les mêmes conditions, nous laissent supposer l'intervention de facteurs de cette nature. Les différences, encore plus grandes, qui existent entre individus de même race dans le même troupeau constituent un autre argument en faveur d'une telle hypothèse : pour les 63 normandes contrôlées, l'écart-type des $t_{c}$ moyens au cours de la lactation est de 4,I2; pour les 44 Frisonnes il est de 3,74, ce qui correspond à des coefficients de variations respectivement de 24,8 et I8,7 p. Ioo. Le $t_{c}$ moyen présente donc une très grande variabilité naturelle (deux à trois fois plus grande que celle du T. B., par exemple).

Quelle est, dans cette variabilité, la part due à des facteurs génétiques ?

Nous avons essayé de répondre à cette question en analysant les résultats du contrôle de 8 paires de jumelles univitellines et de 8 paires de jumelles bivitellines, placées dans des conditions aussi semblables que possible. A partir des courbes individuelles d'évolution $\mathrm{du} t_{c}$ en fonction du stade de lactation, nous avons pu constater qu'il existe, en général, une très grande similitude entre vraies jumelles, alors que, pour les fausses jumelles, les courbes peuvent s'écarter considérablement (figures 4 et 5). Les jumelles univitellines de la figure 4 ayant vêlé à I5 jours d'intervalle, nous avons porté en abscisse non plus les stades de lactation, mais les dates des contrôles : 


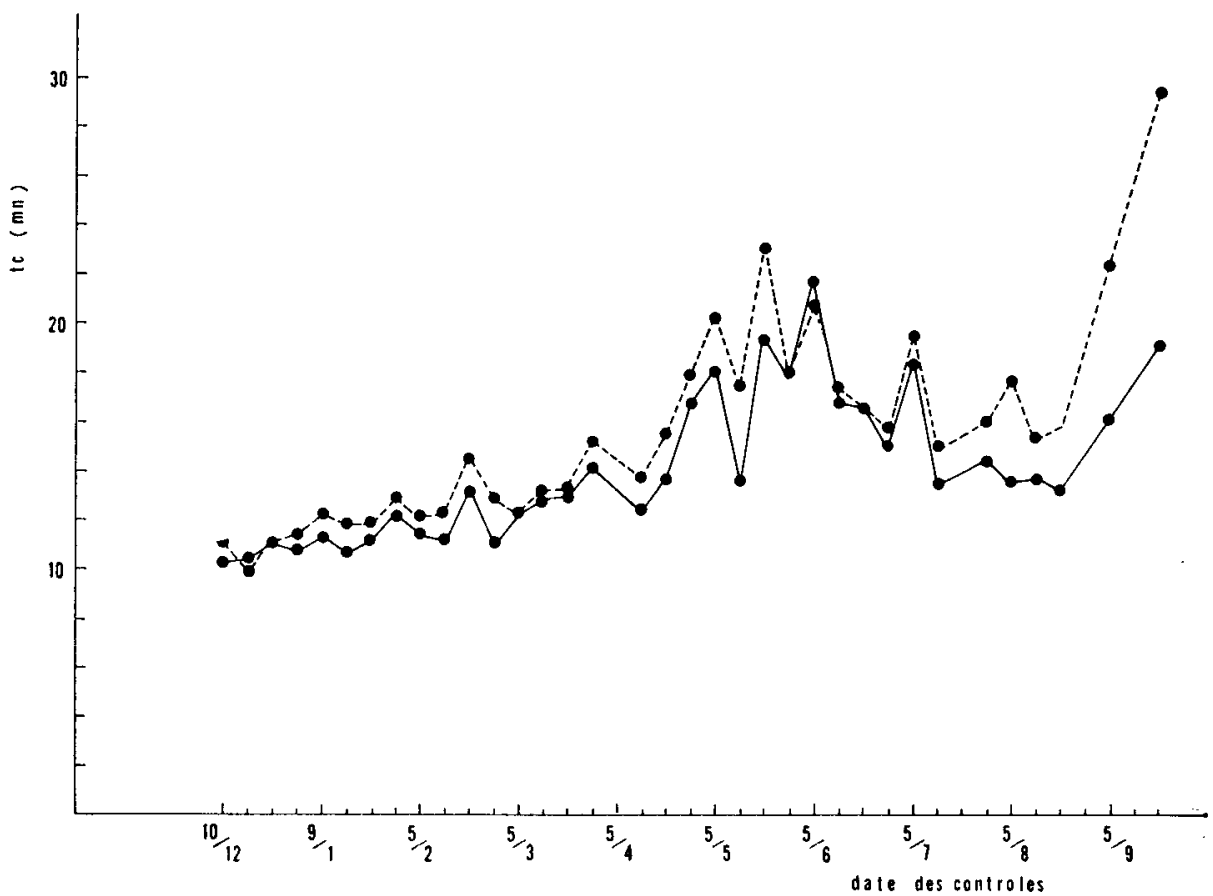

FIG. 4 - Evolution du temps de coagulation avec le stade de laclation : comparaison entre deux jumelles univitellines. L'une des jumelles a vêlé 15 jours après sa soeur : pour éviter un décalage entre les courbes nous avons porté en abscisse les dates des contrôles successifs et non plus les stades de lactation.

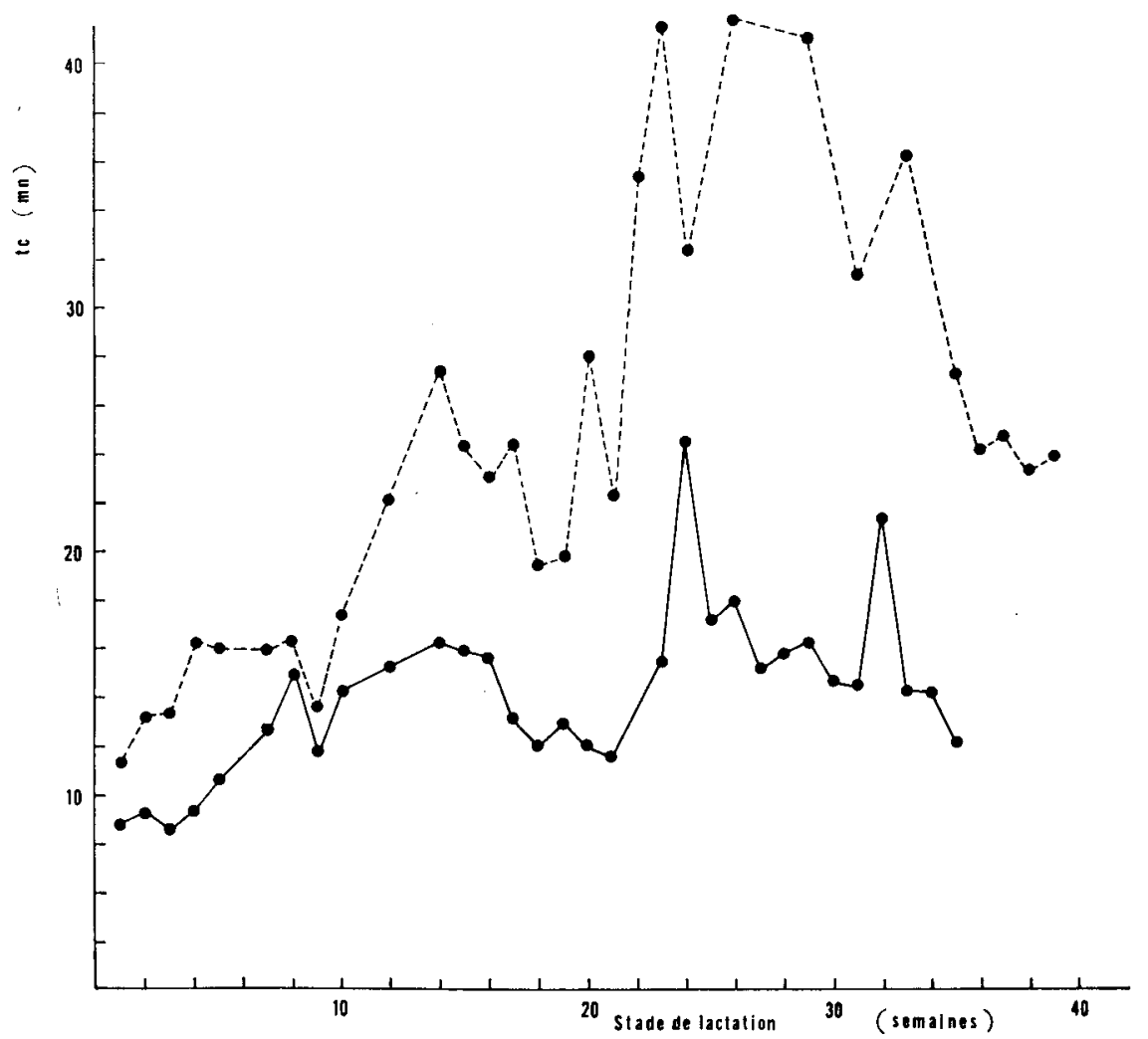

FIG. 5 - Evolution du temps de coagulation avec le stade de lactation : comparaison entre deux jumelles bivitellines, ayant vêlé d̀ 3 jours d'intervalle. 
des contrôles simultanés ont alors la même abscisse. Pour ḷ les fausses jumelles de la figure 5, l'écart entre vêlage n'étant que de 3 jours, nous avons admis qu'elles étaient au même stade de lactation le même jour.

Ia simultanéité des fluctuations hebdomadaires, frappante chez les vraies jumelles, montre qu'il existe un facteur " jour de contrôle " non négligeable, dû à des variations des facteurs d'environnement, soit lors de l'épreuve de coagulation, soit au niveau du troupeau (alimentation, climat,...).

Dans la figure 5, nous avons choisi, à dessein, une paire de fausses jumelles présentant entre elles de gros écarts pour bien montrer l'amplitude qu'ils peuvent prendre. Il faut cependant noter qu'en fait un certain nombre de fausses jumelles montrent, dans cette étude et pour ce caractère, autant de similitude que des vraies jumelles.

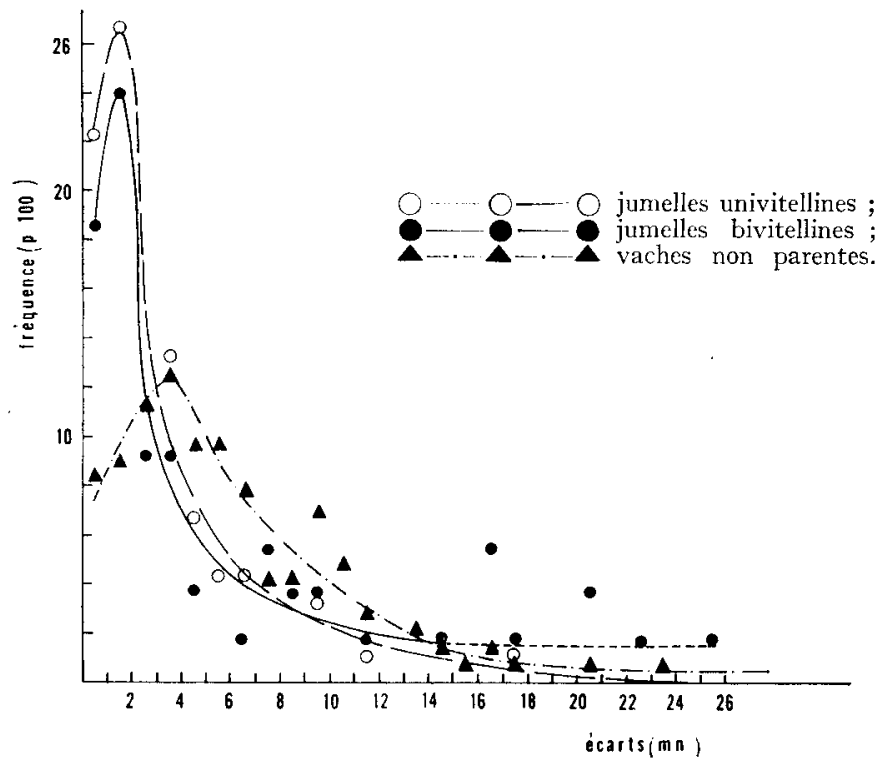

Fig. 6-Distribution des écarts entre temps de coagulation au mème slade de laclation observés sur des couples d'individus de parenté plus ou moins grande.

Ces écarts ont été calculés à partir de courbes ajustées afin d'éliminer les fluctuations journalières.

L'examen du degré de ressemblance entre jumelles a été effectué d'une part sur les $t_{c}$ moyens pour l'ensemble de chaque lactation, d'autre part sur les $t_{c}$ instantanés observés au cours de la lactation.

Les jumelles univitellines et les jumelles bivitellines correspondent respectivement à des coefficients de parenté de $\mathrm{I}$ et 0,5 . Afin d'examiner également le cas où la parenté est nulle, nous avons reconstitué, par tirage au sort parmi l'ensemble des jumelles vraies ou fausses, $x_{5}$ paires artificielles d'individus non parents (le hasard ayant fait qu'une paire de vraies jumelles s'est trouvée ainsi reconstituée, nous l'avons éliminée dans nos calculs).

En ce qui concerne les temps de coagulation moyens, le tableau 2 montre que l'écart moyen entre $t_{c}$ d'individus de la même paire est d'autant plus grand que la parenté est plus faible. 


Alors que les écarts inférieurs à $4 \mathrm{mn}$ correspondent à Ioo p. roo des cas pour les jumelles univitellines, ils ne représentent plus que $50 \mathrm{p}$. Ioo des cas pour les jumelles bivitellines, et $33 \mathrm{p}$. Ioo seulement des cas pour les individus non parents.

Au niveau des $t_{c}$ instantanés, nous retrouvons la même liaison entre degré de ressemblance et parenté des individus d'une même paire ; les écarts entre $t_{c}$ instantanés au cours de la lactation sont en moyenne de 2 , I mn pour les jumelles univitellines, 4,8 $\mathrm{mn}$ pour les jumelles bivitellines et $6, \mathrm{I} \mathrm{mn}$ pour les vaches non parentes. Afin d'éliminer en partie les écarts dus aux fluctuations journalières, nous avons fait la même étude, mais à partir des courbes ajustées et les résultats obtenus ont été du même ordre, soit respectivement $3,2 \mathrm{mn}, 5,9 \mathrm{mn}$ et $5,9 \mathrm{mn}$.

La figture 6 fait apparaître la répartition de ces écarts pour les trois catégories d'animaux. La différence entre jumeaux univitellins et bivitellins, et surtout celle entre jumeaux et individus non parents, ressort nettement, bien que l'écart moyen soit le même entre faux jumeaux et non parents. Le graphique montre également que les distributions des écarts sont tout à fait " anormales "; aussi n'est-il pas possible, du moins sur les données brutes, d'effectuer l'analyse de variance que nous projetions et qui nous aurait permis de chiffrer, pour chaque type de parenté, la variance entre paire, entre stade de lactation et intrapaire.

Cependant, l'ensemble de nos résultats nous permet de conclure que le caractère "temps de coagulation du lait » est en grande partie déterminé par le génotype de 1'individu. Étant donné la très grande variabilité de ce caractère, il doit donc être possible de l'améliorer par sélection. D'autres travaux actuellement en cours nous permettront de préciser ce dernier point.

Reçu en janvier 1960.

\section{SUMMARY}

SOML FACTORS OF VARIATION OF THE RENNETING-TIME OF INDIVIDUAL COWS MILKS

The infuence of different factors on the renneting time was studied from weekly tests on individual milks in the course of 107 lactations $\left(6_{3}\right.$ Normandes $(\mathrm{N})$ and 44 French Friesian (FFPN) .

The B. C. P. rennet test of DAVIS and MCCLEMONT (1939) was used. The main results obtained are:

- Renneting times of heifers are slightly smaller than those of cows in their second and third lactation.

- The stage of lactation has a marked influence on the renneting time $t_{c}:$ after a phase of increase spread out over the first zoo days of lactation, a maximum is reached, which is only slightly marked, and then a gentle decrease during the last roo days of lactation. Actually, $t_{c}$ varies, on the average, from 75 to I $_{5}$, if one assumes its mean value throughout the whole of the lactation period is equal to roo.

- The general trend of the renneting time with the stage of lactation seems to be largely related to the variation of the calcium/total nitrogen ratio in the milk.

- Important sources of variation are the breed and, even more, the individual cow : the average renneting time for the whole lactation is $16,6 \pm 0,5 \mathrm{mn}$ for the Normandes and $20,0 \pm 0,6 \mathrm{mn}$ for the FFPN. Even in the same herd the coefficients of variation of the average renneting times are very high : 24,8 p. Ioo for Normandes and i $8,7 \mathrm{p}$. Ioo for Friesian.

- The analysis of the results obtained from 8 pairs of monozygotic twins and 8 pairs of dizygotic twins clearly shows that the character " rate of coagulation " of the milk is partly dependent on genetic factors. Refering to the great variability of this characteristic, it should be possible, to improve it by selection.

\section{REMERCIEMENTS}

Nous remercions M. G. MocQuot qui est à l'origine de ce travail et dont les conseils nous ont été très précieux, M. C. AlaIs qui nous a permis de mettre au point la technique opératoire et qui a titré périodiquement notre solution de présure ainsi que M. M. PLommet qui nous a transmis les 
résultats des examens bactériologiques détaillés qu'il a effectués régulièrement sur les laits de toutes les vaches utilisés dans cette étude.

\section{RÉFÉRENCES BIBLIOGRAPHIQUES}

Auriol P., 1954. Possibilités d'amélioration, par la sélection, de la production fromagère de la race pierouge de l'Est. C.R.Acad. Agr. Fr., 40,332-337.

Auriol P., Mocouot G. 1957. Données non publiées.

Davis J. G., MeCenmont J., I 939. Studies in mastitis, J. Dairy Res., 10, 94-107.

JENNESS R., I953. Titration of calcium and macrnesium in milk and milk fractions with ethylenediamine tetracetate. Analyt. Chem., 25, 966-968.

Koestler A. G., I925. Different types of milk, their relations to the renuet and their importance in cheese making. J. Dairy Sci., 8, 28-36.

LiNG E. R., 19.37. The composition of milk and whey. J. Dairy Res., 8, I73-193.

McDowald F. H., Dolby R. M., McDowei.l. A. K. R., 1937. 'The coagulation of milk with rennet. $J$. Dairy Res., 8, 31-52.

Mocquot G., Alais C., Chevaljer R., I954. Étude sur les défauts de coagulation du lait par la présure. Ann. Techn. Agric., 1, I-44.

Peltola L., I949. The coagulation of milk of the Finnish cows with rennet. XXII0 Congr. Int. Laiterie, 2, $73-80$.

PELTOLA E., I957. Die Iabgerimung der Milch mit besonderer Berücksichtigung der sog. Iabträgen Milch. Milchizsensch., $12,473-476$.

Peltola E., VoGT P., r959. The effect of cold ageing on the remeting of milk. Ini. Dainy Congr., 1, $268-27$ I. 